\title{
High and Low Type One-dimensional Function with Multi-Step Uniform Invariant Density
}

\author{
Toshihiko Yasuda \\ Dept. of Mechanical System Engineering \\ The University of Shiga Prefecture, \\ 2500 Hassaka-cho, Hikone, Shiga, 522-8533, Japan \\ E-mail: yasuda@mech.usp.ac.jp
}

\begin{abstract}
Nonlinear systems described by simple mathematical models often exhibit extremely complicated behavior called chaos. In this paper, chaotic behavior, exhibited by the one-dimensional difference equation, is investigated. A method for constructing nonlinear functions with the invariant density is newly demonstrated. Furthermore, the method for finding the invariant density of the nonlinear function is also presented. Nonlinear functions, treated in this study, are piecewise linear ones and the invariant density is piecewise uniform.
\end{abstract}

\section{Introduction}

In various research fields, it is well known that nonlinear systems with a simple mathematical model often exhibit extremely complicated behavior called chaos [1-12]. In this paper, chaotic behavior, exhibited by the following deterministic system described by the one-dimensional difference equation, is discussed:

$$
\begin{gathered}
x_{n+1}=f\left(x_{n}\right), \quad n=0,1,2, \cdots, \\
f: I \rightarrow I, I=[0,1] .
\end{gathered}
$$

Concerned with the nonlinear function $f(x)$, which determines the characteristic of the nonlinear system (1), if a function $p(x), x \in[0,1]$ satisfies the following Frobenius-Perron equation

$$
p(y)=\sum_{x \in f^{-1}(y)} \frac{p(x)}{\left|f^{\prime}(x)\right|}, \quad f^{\prime}(x)=\frac{d f(x)}{d x},
$$

then the function $p(x)$ is called the invariant density of the nonlinear function $f(x)$. Furthermore, if the invariant density $p(x)$ satisfies the following normalized condition

$$
\int_{I} p(x) d x=1
$$

then we especially call the function $p(x)$ as the invariant probability density of the nonlinear function $f(x)$. Important statistical information of chaotic behavior of the system (1) are included in the invariant probability density $p(x)$. For example, for any subset $A \subset R^{1}$, the equation

$$
\lim _{n \rightarrow \infty} \frac{1}{n} \sum_{k=0}^{n-1} \chi_{A}\left(x_{k}\right)=\int_{f} \chi_{A}(x) p(x) d x
$$

holds, where

$$
\chi_{A}(x)=\left\{\begin{array}{ll}
1, & x \in A \\
0, & x \notin A
\end{array} .\right.
$$

The equation (3) indicates that the invariant density gives the frequency that solution processes $\left\{x_{n}, n=0,1,2, \cdots\right\}$ visit a set $A$. Namely, the invariant density of chaotic system is equivalent to the stationary probability density function of stochastic systems. For example, it is well-known that Tent Map has invariant density with uniform distribution [5]. Nonlinear systems described by nonlinear map with invariant density can be used as pseudorandom number generators $[9,13,14]$. Recently, there exists a trial to utilize chaotic sequences, generated by one-dimensional piecewise linear maps with uniform invariant density, for built-in self-test of LSI [16]. As mentioned above, theoretical studies and applications of chaos are significant from both scientific and engineering viewpoints and there exist many researches relevant to chaos [15-19]. The invariant density of deterministic chaotic systems is extremely interesting and is the first step of research on statistical aspect of chaos. Therefore, studies on the invariant density of simple dynamical systems are important in theory and in practice. In previous work, a class of chaotic systems, whose invariant density is the piecewise uniform distribution, was demonstrated $[20,21]$. In this investigation, a class of nonlinear discrete system with the invariant density, which is multi-step type piece-wise uniform, is newly introduced. Results, presented in this paper, are a generalization of previous investigations $[20,21]$, concerned with piecewise linear functions with the piecewise uniform invariant probability density. 


\section{Method for Constructing Piecewise Linear Function with Invariant Measure}

In this section, we show the method for constructing piecewise linear type nonlinear function with the invariant density.

(Step 1) A interval $[0,1]=\{x: 0 \leq x \leq 1\}$ is divided into $n$ intervals with dividing points $0=p_{0}<p_{1}<\cdots<p_{n}=1$. The following intervals are defined as

$$
\begin{aligned}
& I_{1}=\left[0, p_{1}\left[=\left[p_{0}, p_{1}\left[=\left\{x: 0=p_{0} \leq x<p_{1}\right\},\right.\right.\right.\right. \\
& I_{2}=\left[p_{1}, p_{2}\left[=\left\{x: p_{1} \leq x<p_{2}\right\},\right.\right. \\
& \quad \vdots \\
& I_{n-1}=\left[p_{n-2}, p_{n-1}\left[=\left\{x: p_{n-2} \leq x<p_{n-1}\right\},\right.\right. \\
& I_{n}=\left[p_{n-1}, 1\right]=\left[p_{n-1}, p_{n}\right]=\left\{x: p_{n-1} \leq x \leq p_{n}=1\right\} .
\end{aligned}
$$

(See Fig.1(a))

(Step 2) As shown in Fig.1(b), we draw vertical lines through points $\left(p_{i}, 0\right) i=1,2, \cdots, n$ and horizontal lines through points $\left(0, p_{i}\right) i=1,2, \cdots, n$. In this step, many rectangles are created in the area $\{(x, y): 0 \leq x \leq 1,0 \leq y \leq 1\}$.

(Step 3) For convenience of explanations, in this paper, it is assumed that,

(A1) On each interval $I_{i}$ included in $x$-axis, there exists only one segment, which construct piecewise linear function.

First, on the interval $I_{n}$, we draw a segment with terminal points $\left(p_{n-1}, p_{n-1}\right)$ and $\left(p_{n}, 0\right)$. (See Fig.1(c))

(Step 4) On remained intervals $I_{i}, i=1,2, \cdots, n-1$, we draw segments and we yield a piecewise linear function $f(x)$ according to following manners (See Fig.1(d)):

(C1) The nonlinear function $f(x)$ is a mapping from an interval $I_{i}$ onto another interval $I_{j}$ satisfying that

$$
f\left(I_{i}\right)=I_{j}, \quad i=1,2, \cdots, n-1, \quad j=1,2, \cdots, n .
$$

with $f\left(I_{i}\right) \neq I_{i}$, where $f\left(I_{n}\right)=\left\{f(x): x \in I_{n}\right\}$. Each segment is a diagonal line of a rectangle, constructed in previous Step 2.

$$
f\left(I_{n}\right)=I_{1} \cup I_{2} \cup \cdots \cup I_{n-1}
$$

$$
\bigcup_{k=1}^{n-1} f^{-k}\left(I_{n}\right)=I_{1} \cup I_{2} \cup \cdots \cup I_{n-1},
$$

where $f^{-1}\left(I_{i}\right)=\left\{x: f(x)=I_{j}\right\}$.

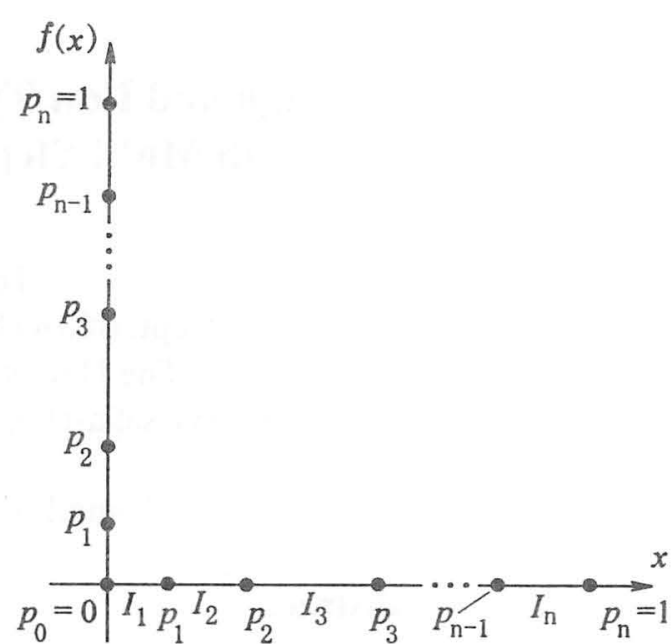

(a) Step 1

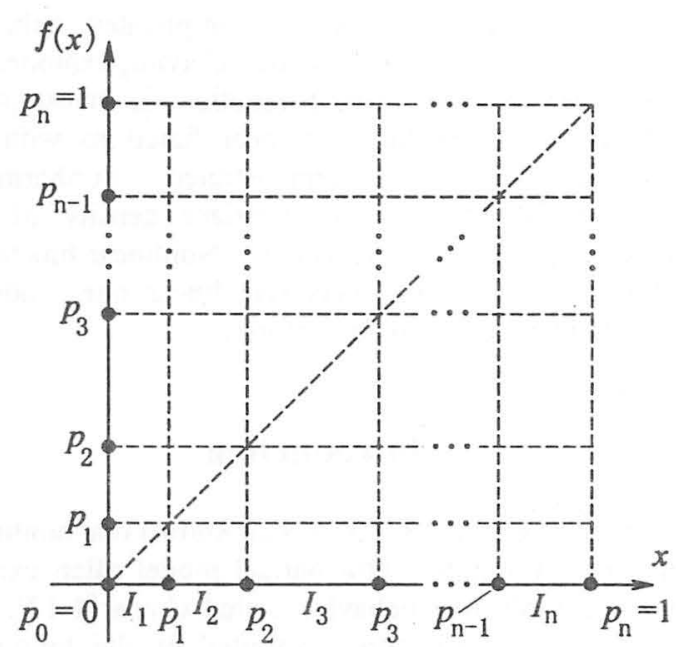

(b) Step 2

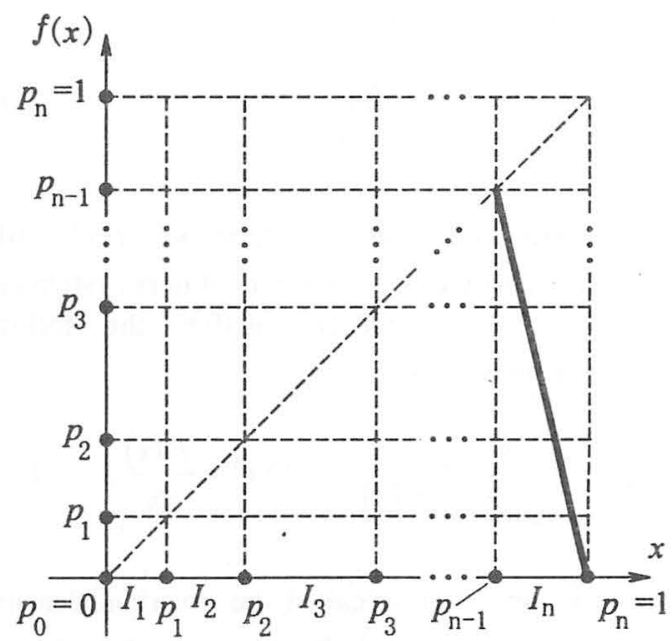

(c) Step 3

Fig.1 Steps constructing a piecewise linear function 


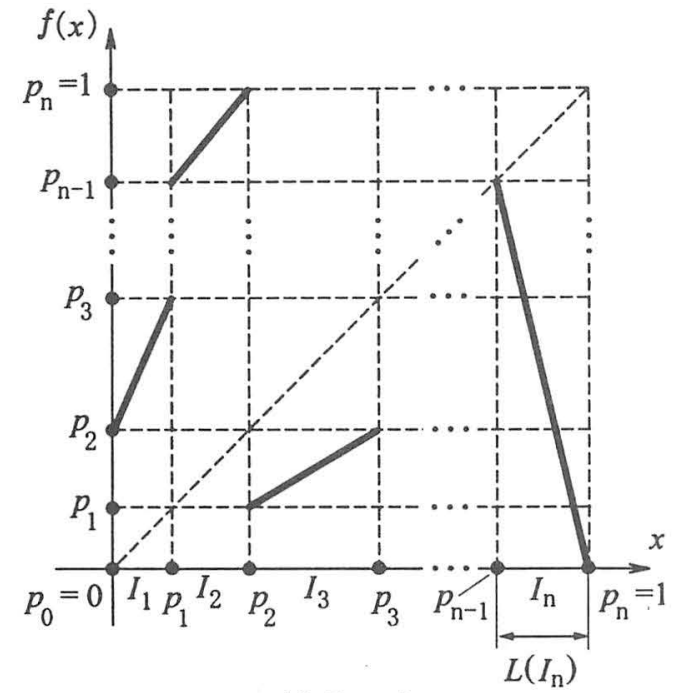

(d) Step 4

Fig.1 Steps constructing a piecewise linear function (continued)

\section{Method for Finding Invariant Density}

In this section, the method for finding the invariant density $p(x)$ of the nonlinear function $f(x)$ constructed in Sec. 2 is shown.

(Step 1) Assume that, a candidate of the invariant density $p(x)$ satisfies that

$$
p(x)=1, \quad x \in I_{n} .
$$

(Step 2) Note that, from an assumption (A1) and from (Step 3) in Sec.2 and conditions (C1) to (C3), if $y \in I_{n}$, then $f^{-1}(y)$ is a point satisfying that

$$
f^{-1}(y) \in f^{-1}\left(I_{n}\right)
$$

Appling Frobenius-Perron equation (2) to any point $y \in I_{n}$ and using Eq.(6), we obtain

$$
1=p(y)=\sum_{x \in f^{-1}(y)} \frac{p(x)}{\left|f^{\prime}(x)\right|}=\frac{p(x)}{\frac{L\left(I_{n}\right)}{L\left(f^{-1}\left(I_{n}\right)\right)}}, \text { (7) }
$$

where $L\left(I_{i}\right)$ is the length of the interval $I_{i}$, namely,

$$
L\left(I_{i}\right)=p_{i}-p_{i-1}
$$

(See Fig.1(d)) Remembering that $L\left(I_{j}\right)$ is the length of the interval $I_{j}$ and referring Fig.2, it is easy to obtain that, if $y \in I_{n}$ and $y \in I_{n}$, then

$$
\left|f^{\prime}(x)\right|=\frac{L\left(I_{n}\right)}{L\left(f^{-1}\left(I_{n}\right)\right)} \text {. }
$$

From Eq.(7), we obtain

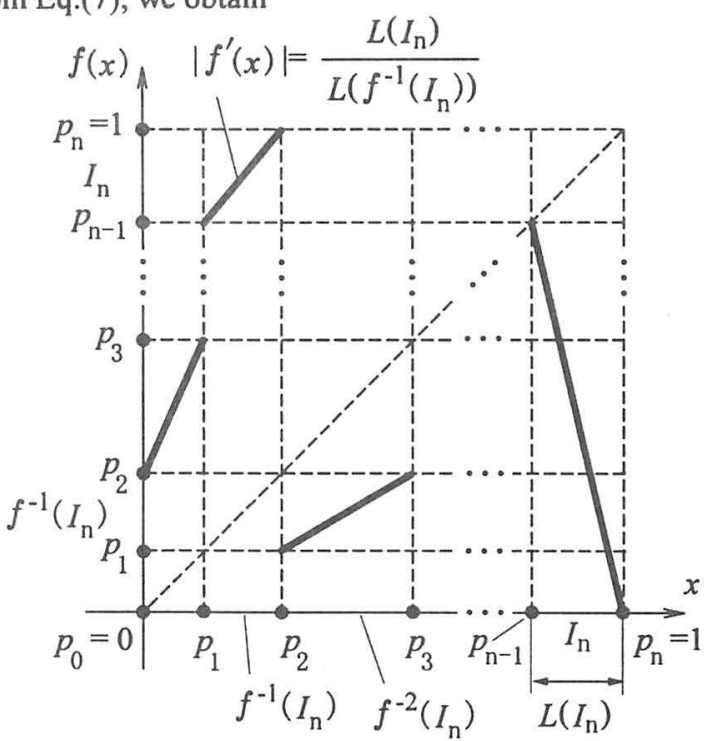

Fig.2 Finding process of the invariant density

$$
p(x)=\frac{L\left(I_{n}\right)}{L\left(f^{-1}\left(I_{n}\right)\right)}, \quad x \in f^{-1}\left(I_{n}\right) .
$$

(Step 3) Note that, under assumption (A1) and conditions (C1) to (C3), if $y \in f^{-1}\left(I_{n}\right)$, then

$$
f^{-1}(y)=\left\{x_{1}, x_{2}: x_{1} \in f^{-2}\left(I_{n}\right), x_{2} \in I_{n}\right\}
$$

(See Fig.2) Appling Frobenius-Perron equation (2) to any point $y \in f^{-1}\left(I_{n}\right)$, we obtain

$$
p(y)=\frac{p\left(x_{1}\right)}{\frac{L\left(f^{-1}\left(I_{n}\right)\right)}{L\left(f^{-2}\left(I_{n}\right)\right)}}+\frac{p\left(x_{2}\right)}{\frac{1-L\left(I_{n}\right)}{L\left(I_{n}\right)}} .
$$

Noting that $y \in f^{-1}\left(I_{n}\right)$ and $x_{2} \in I_{n}$, from Eqs.(6) and (8), we obtain that, in Eq.(9),

$$
\begin{aligned}
& p(y)=\frac{L\left(I_{n}\right)}{L\left(f^{-1}\left(I_{n}\right)\right)}, \\
& p\left(x_{2}\right)=1 .
\end{aligned}
$$

Substituting these equation into Eq.(9), we obtain

$$
\begin{aligned}
p\left(x_{1}\right) & =\frac{L\left(f^{-1}\left(I_{n}\right)\right)}{L\left(f^{-2}\left(I_{n}\right)\right)}\left(\frac{L\left(I_{n}\right)}{L\left(f^{-1}\left(I_{n}\right)\right)}-\frac{L\left(I_{n}\right)}{1-L\left(I_{n}\right)}\right) \\
& =\frac{L\left(I_{n}\right)}{L\left(f^{-2}\left(I_{n}\right)\right)}\left(\frac{1-L\left(I_{n}\right)-L\left(f^{-1}\left(I_{n}\right)\right)}{1-L\left(I_{n}\right)}\right) . \\
& =\frac{L\left(I_{n}\right)}{1-L\left(I_{n}\right)} \cdot \frac{1-L\left(I_{n}\right)-L\left(f^{-1}\left(I_{n}\right)\right)}{L\left(f^{-2}\left(I_{n}\right)\right)}
\end{aligned}
$$


$x_{1} \in f^{-2}\left(I_{n}\right)$.

(Step 4) Similarly, if $y \in f^{-2}\left(I_{n}\right)$, then

$$
f^{-1}(y)=\left\{x_{1}, x_{2}: x_{1} \in f^{-3}\left(I_{n}\right), x_{2} \in I_{n}\right\} .
$$

Hence, we obtain

$$
p(y)=\frac{p\left(x_{1}\right)}{\frac{L\left(f^{-2}\left(I_{n}\right)\right)}{L\left(f^{-3}\left(I_{n}\right)\right)}}+\frac{p\left(x_{2}\right)}{\frac{1-L\left(I_{n}\right)}{L\left(I_{n}\right)}} .
$$

From Eqs.(6) and (10), we obtain

$$
p\left(x_{1}\right)=\frac{L\left(I_{n}\right)}{1-L\left(I_{n}\right)} \cdot \frac{1-L\left(I_{n}\right)-L\left(f^{-1}\left(I_{n}\right)\right)-L\left(f^{-2}\left(I_{n}\right)\right)}{L\left(f^{-3}\left(I_{n}\right)\right)},
$$

Repeating above process, we obtain that, on any interval $r$, $k=2,3,\ulcorner, \mathrm{n}-1$, the invariant density is given by

$$
p(x)=\frac{L\left(I_{n}\right)}{1-L\left(I_{n}\right)} \cdot \frac{1-\sum_{k=0}^{k-1} L\left(f^{-k}\left(I_{n}\right)\right)}{L\left(f^{-k}\left(I_{n}\right)\right)} .
$$

The invariant density $p(x)$ is normalized by

$$
p_{\text {norm }}(x)=\frac{p(x)}{\int p(x) d x} .
$$

\section{Examples}

In order to show the validity of results demonstrated in previous chapter, three illustrative examples are demonstrated here.

\subsection{Example 1}

First, we show the nonlinear system, which is slightly modified example of previous one, presented in Ref.[21]. This system has three dividing points

$$
p_{1}=0.1, p_{2}=0.3 \text { and } p_{3}=0.7 .
$$

The difference equation and the piecewise linear function $f_{1}(x)$ is given by

$$
\begin{aligned}
& x_{n+1}=f_{1}\left(x_{n}\right), \\
& f_{1}(x)=\left\{\begin{array}{lr}
2 x+0.1, & 0 \leq x<0.1 \\
2 x+0.1, & 0.1 \leq x<0.3 \\
\frac{3}{4} x+\frac{19}{40}, & 0.3 \leq x<0.7 \\
-\frac{7}{3} x+\frac{7}{3}, & 0.7 \leq x \leq 1
\end{array}\right.
\end{aligned}
$$

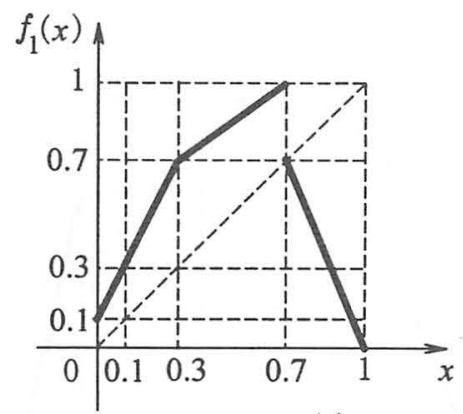

Fig.3 Nonlinear function $f_{1}(x)$ of example 1

Figure 3 shows the shape of the nonlinear function $f_{1}(x)$. The invariant probability density of the nonlinear function $f_{1}(x)$ is given by

$$
\widetilde{p}_{1}(x)=\left\{\begin{array}{cc}
\frac{5}{9}, & 0 \leq x<0.1 \\
\frac{5}{6}, & 0.1<x<0.3 \\
\frac{35}{36}, & 0.3<x<0.7 \\
\frac{35}{27}, & 0.7<x \leq 1
\end{array} .\right.
$$

The shape of the invariant probability density $\widetilde{p}_{1}(x)$ of the nonlinear function $f_{1}(x)$ and a solution process are plotted in Fig.4.

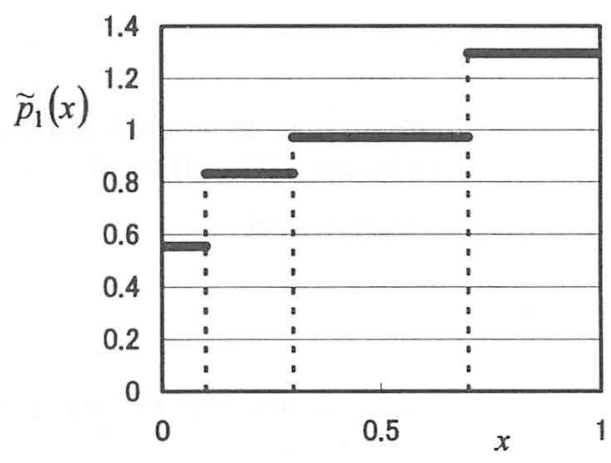

(b) The invariant density $\tilde{p}_{1}(x)$

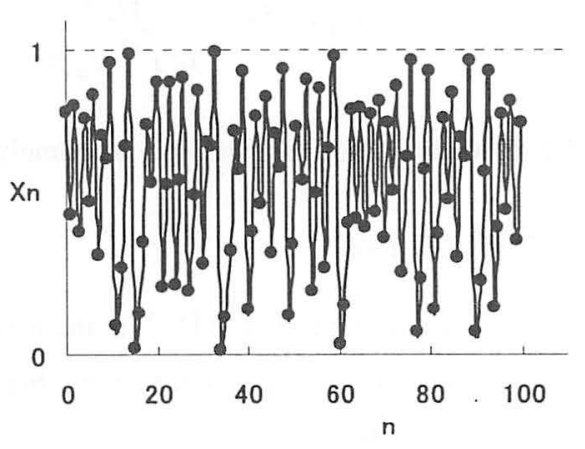

(b) A solution process

Fig.4 Some characteristics of the system described by the nonlinear function $f_{1}(x)$ 


\subsection{Example 2}

Secondly, we consider the 2nd nonlinear system, which is also three dividing points $p_{1}=0.1, p_{2}=0.3$ and $p_{3}=0.7$. The difference equation and the piecewise linear function $f_{2}(x)$ is given by

$$
\begin{aligned}
& x_{n+1}=f_{2}\left(x_{n}\right), \\
& f_{2}(x)=\left\{\begin{array}{lc}
4 x+0.3, & 0 \leq x<0.1 \\
\frac{3}{2} x+\frac{11}{20}, & 0.1 \leq x<0.3 \\
\frac{1}{2} x-\frac{1}{20}, & 0.3 \leq x<0.7 \\
-\frac{7}{3} x+\frac{7}{3}, & 0.7 \leq x \leq 1
\end{array} .\right.
\end{aligned}
$$

Figure 5 shows the shape of the nonlinear function $f_{2}(x)$. The invariant probability density $\tilde{p}_{2}(x)$ of the nonlinear function $f_{2}(x)$ is given by

$$
\tilde{p}_{2}(x)=\left\{\begin{array}{cc}
\frac{15}{23}, & 0 \leq x<0.1 \\
\frac{105}{46}, & 0.1<x<0.3 \\
\frac{75}{92}, & 0.3<x<0.7 \\
\frac{35}{23}, & 0.7<x \leq 1
\end{array} .\right.
$$

The shape of the invariant probability density $\tilde{p}_{2}(x)$ of the nonlinear function $f_{2}(x)$ is plotted in Fig.6.

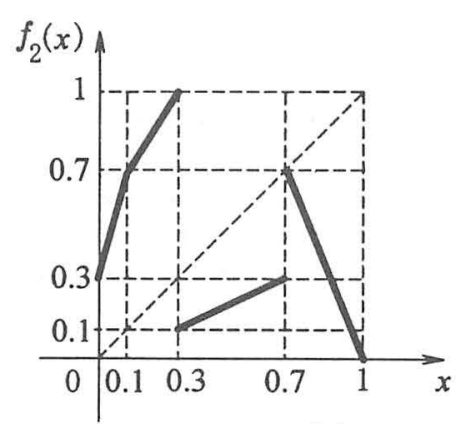

Fig.5 Nonlinear function $f_{2}(x)$ of example 2

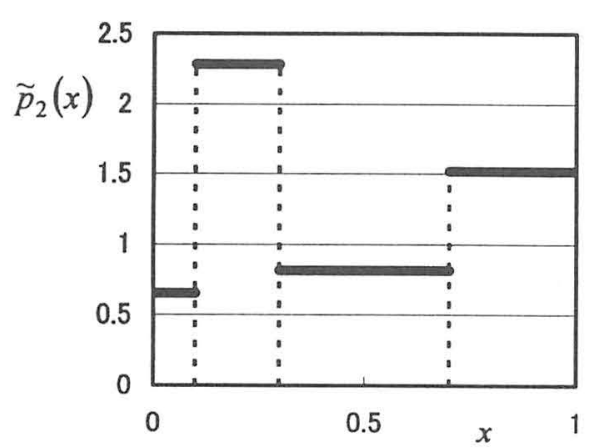

Fig.6 The invariant density $\tilde{p}_{2}(x)$ of the nonlinear function $f_{2}(x)$

\subsection{Example 3}

Final example is described by the following difference equation and the piecewise linear function $f_{3}(x)$

$$
\begin{aligned}
& x_{n+1}=f_{3}\left(x_{n}\right), \\
& f_{3}(x)=\left\{\begin{array}{lr}
3 x+0.7, & 0 \leq x<0.1 \\
2 x+0.1, & 0.1 \leq x<0.3 \\
\frac{1}{4} x-\frac{3}{40}, & 0.3 \leq x<0.7 \\
-\frac{7}{3} x+\frac{7}{3}, & 0.7 \leq x \leq 1
\end{array}\right.
\end{aligned}
$$

Figure 7 shows the shape of the nonlinear function $f_{3}(x)$.

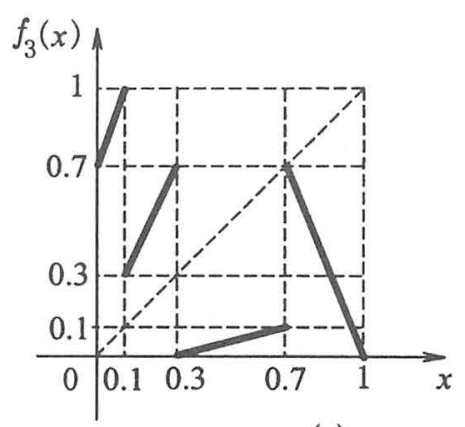

Fig.7 Nonlinear function $f_{3}(x)$ of example 3

The invariant probability density $\tilde{p}_{3}(x)$ of the nonlinear function $f_{3}(x)$ is given by

$$
\tilde{p}_{3}(x)=\left\{\begin{array}{cc}
\frac{5}{9}, & 0 \leq x<0.1 \\
\frac{5}{6}, & 0.1<x<0.3 \\
\frac{35}{36}, & 0.3<x<0.7 \\
\frac{35}{27}, & 0.7<x \leq 1
\end{array} .\right.
$$

The shape of the invariant probability density $\tilde{p}_{3}(x)$ of the nonlinear function $f_{3}(x)$ is plotted in Fig.8.

These three examples show the validity of the results demonstrated in this investigation.

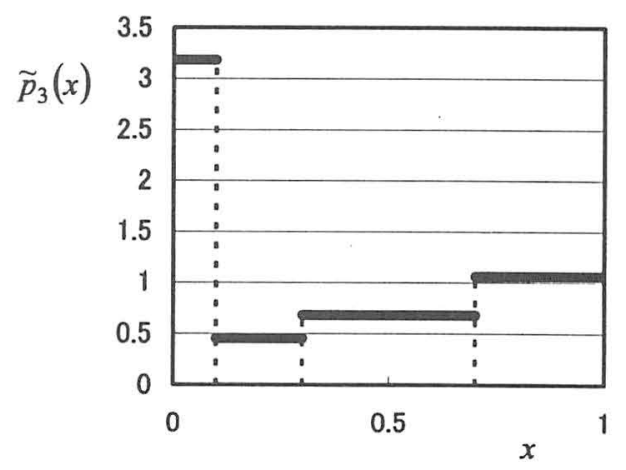

Fig. 8 The invariant density $\tilde{p}_{3}(x)$ of the nonlinear function $f_{3}(x)$ 


\section{Conclusions}

In this paper, chaotic behavior, exhibited by the one-dimensional difference equation, has been discussed. Methods for constructing nonlinear functions and finding the invariant density are demonstrated. Some nonlinear discrete systems with the invariant density, which is multistep type piece-wise uniform, have been newly introduced. Numerical studies show the validity of this study. In previous results, the position of segments constructing the nonlinear function on intervals $I_{j}, \quad i=1,2, \cdots, n-1$ uniformly climb upward. Nonlinear functions, introduced in this studies, has up and down type shapes.

\section{References}

[1] T. Y. Li. and J. A. Yorke, Period three implies chaos, Amer. Math. Mon., vol.82, pp.985-992, 1977.

[2] R. May, Simple mathematical modes with very complicated dynamics, Nature, vol.261, pp.459-467, 1981.

[3] R. L. Devaney, An Introduction to Chaotic Dynamical Systems, Addison Wesley, Redwood City, 1989.

[4] H. G. Schuster, Deterministic Chaos - An Introduction, 2nd ed., VCH Verlagsgesellschaft Weinheim, 1989.

[5] F. C. Moon, Chaotic and Fractal Dynamics - An Introduction for Applied Scientists and Engineers, John Wiley \& Son, 1992.

[6] A. Lasota and M. C. Mackey, Chaos, Fractals, and Noise - Stochastic Aspects of Dynamics -, Springer-Verlag New York, 1994.

[7] R. C. Hilborn, Chaos and Nonlinear Dynamics - An Introduction for Scientists and Engineers -, Oxford Univ. Press, 2000.

[8] G. Chen, Controlling Chaos and Bifurcation in Engineering Systems, CRC Press, 2000

[9] W. Steeb, The Nonlinear Workbook, 3rd ed., World Scientific Pub., 2005.

[10] M. V. Jakoson, Absolutely continuous invariant measures for one-parameter families of one-dimensional families of one-dimensional maps, Commun. Math. Phys., Vol.81, pp.39-88, 1981

[11] A. Lasota and J. A. York, On the existence of invariant measure for piecewise monotonic transformations (1), Trans. Am. Math. Soc., Vol.186, pp.481-488, 1973.

[12] T. Kohda and K. Aihara, Chaos in discrete systems and diagnosis of experimental chaos, Trans. IEICE Jpn., Vol.E73, No.6, pp.772-783, 1990.

[13] T. Y. Li and J. A. York, Ergodic transformations from an interval into itself, Trans. Am. Math., vol.235, pp183-192, 1978.

[14] T. Y. Li and J. A. York, Ergodic maps on [0,1] and nonlinear pseudorandom number generators,
Nonlinear Anal., vol.2, pp.473-481, 1978.

[15] Y. Shimizu, M. Miyazaki, H. Lee and K. Akizuki, Chaos synchronization based on fuzzy model using sliding mode control, International Journal of Innovative Computing, Information and Control, Vol.1, No.3, pp.563-579, 2005.

[16] A. Izukura, R. Tsuchida, K. Kudou, D. Yoshioka, A. Tsuneda and $T$. Inoue, $A$ study on test pattern generation for LSI Tests using chaotic sequences, The Institute of Electronics, Information and Communication Engineers Technical Report NLP2006-1, pp.1-4, 2006.

[17] Q. Ding, J. Pang, J. Fang and X. Peng, Designing of chaotic system output sequence circuit based on FPGA and its applications in network encryption card, International Journal of Innovative Computing, Information and Control, Vol.3, No.2, pp.449-456, 2007. Volume 3, Number 3, June 2007

[18] J. Liu, J. Lu, Y. Shi, X. Li and Q. Tang, Different type of synchronization phenomena in unidirectional coupled unified chaotic systems, International Journal of Innovative Computing, Information and Control, Vol.3, No.3, pp.697-708, 2007.

[19] L. Wang, N. Wang and Z. Chu, Almost shift invariant set and distributional chaos in a sequence, International Journal of Innovative Computing, Information and Control, Vol.4, No.8, pp.1853-1859, 2008.

[20] T. Yasuda, On a One-dimensional Chaotic Discrete Dynamical System with Piecewise Uniform Invariant Density, International Journal of Innovative Computing, Information and Control, Vol.4, No.1, pp.143-152, 2007.

[21] T. Yasuda: On a class of one-dimensional chaotic discrete dynamical system with multi step piecewise uniform invariant density, Proceedings of the 40th ISCIE International Symposium on Stochastic Systems Theory and its Applications, pp.288-293 (2009) 